\title{
Combining 3-D geovisualization with force feedback driven user interaction
}

\author{
Adam Faeth \\ Michael Oren \\ Chris Harding \\ Virtual Reality Applications Center (VRAC), lowa State University, Ames, lowa \\ adfaeth@iastate.edu \\ moren@iastate.edu \\ charding@iastate.edu
}

\begin{abstract}
We describe a prototype software system for investigating novel human-computer interaction techniques for 3-D geospatial data. This system, M4-Geo (Multi-Modal Mesh Manipulation of Geospatial data), aims to provide a more intuitive interface for directly manipulating 3-D surface data, such as digital terrain models (DTM). The M4-Geo system takes place within a 3-D environment and uses a Phantom haptic force feedback device to enhance 3-D computer graphics with touch-based interactions. The Phantom uses a 3-D force feedback stylus, which acts as a virtual "finger tip" that allows the user to feel the shape (morphology) of the terrain's surface in great detail. In addition, it acts as a touch sensitive tool for different GIS tasks, such as digitizing (draping) of lines and polygons directly onto a 3-D surface and directly deforming surfaces (by pushing or pulling the stylus in or out). The user may adjust the properties of the surface deformation (e.g., soft or hard) locally by painting it with a special "material color."

The overlap of visual and force representation of 3-D data aides hand-eye coordination for these tasks and helps the user to perceive the 3-D spatial data in a more holistic, multi-sensory way. The use of such a 3-D force feedback device for direct interaction may thus provide more intuitive and efficient alternatives to the mouse and keyboards driven interactions common today, in particular in areas related to digital landscape design, surface hydrology and geotechnical engineering.
\end{abstract}

\section{Categories and Subject Descriptors}

J.2 [PHYSICAL SCIENCES AND ENGINEERING]; I.3.2 Graphics Systems

\section{General Terms}

Design, Human Factors

\section{Keywords}

GIS, geovisualization, 3-D, scientific visualization, spatial analysis, data exploration spatial decision support, humancomputer interaction, virtual environments, haptics

Permission to make digital or hard copies of all or part of this work for personal or classroom use is granted without fee provided that copies are not made or distributed for profit or commercial advantage, and that copies bear this notice and the full citation on the first page. To copy otherwise, to republish, to post on servers or to redistribute to lists, requires prior specific permission and/or a fee.

ACM GIS '08, November 5-7, 2008. Irvine, CA, USA

(c) 2008 ACM ISBN 978-1-60558-323-5/08/11...\$5.00

\section{INTRODUCTION}

\subsection{Motivation}

Geovisualization $[12,16]$ may be described as the intersection of many different fields, including Geographic Information Systems (GIS), 3-D scientific visualization, computer graphics, human-computer interaction and virtual reality.

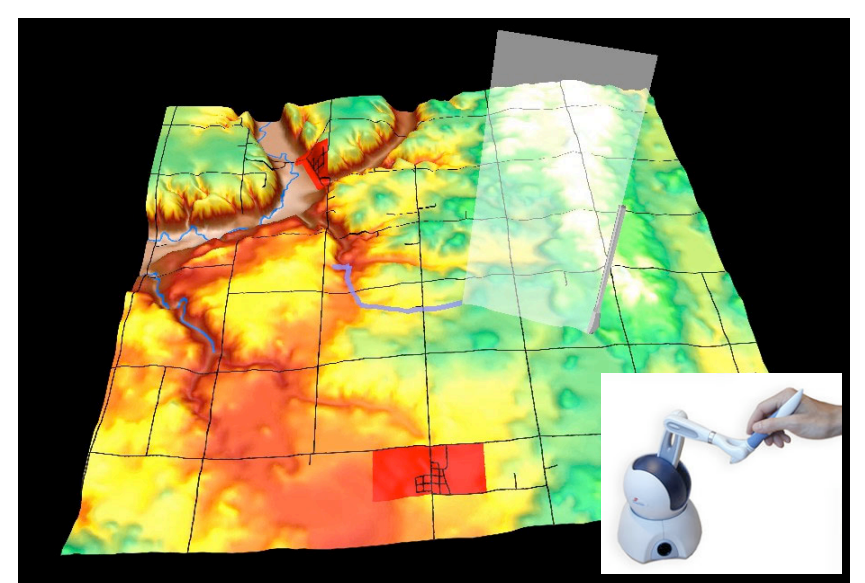

Figure 1. Using the Phantom haptic device, the user is able to feel the terrain's 3-D shape while draping a line feature directly onto a DTM.

The long-term aim of this research is to fundamentally improve the way GIS users are able to interact with 3-D geospatial data by combining 3-D computer graphics with the emerging field of haptics. The use of a stylus as physical interface for user interaction allows us to explore ways of extending the traditional "pencil \& paper map" interface into 3-D and to create a touchable 3-D version of the 2-D paper map. The ability to "trace" the underlying terrain morphology in 3-D is a valuable sensory addition during many types of geoscientific tasks, including the precise placement of points, lines or polygons (figure 1), or the selection of parts of the terrain via painting them with a certain color. Beyond the hapto-visual perception of static geometry, where the user perceives multiple channels of information (output) about a 3-D model but does not change it, the system also supports dynamic, touch-based interactions, such as the real-time terrain deformation, in which the 3-D model changes according to the user's input and the changes can be seen and felt immediately.

Given the ongoing trend from 2-D GIS to (visual) 3-D geovisualization and the spreading use of the phantom in other, 
non-geoscience applications areas, such as 3-D scientific visualization of high-dimensional data or for medical simulation, the specific research questions for our work are: What geoscientific tasks can be fundamentally improved by adding haptics? What technology gives programmers the ability to create visual-haptic 3-D interfaces at a high level and supports several types of common haptic devices? The M4-Geo, multisensory (multimodal) interface presented here, provides several examples of interacting with 3-D geospatial data via both vision and touch. M4-Geo is based on the open source H3D API and offers a view ahead to new types of fundamentally different interactions that would become possible in a next-generation, touch-enabled 3-D GIS system.

\subsection{Related work}

The research presented here relates to geographic visualization [18], especially the 3 -D visualization of geospatial data [29, 4, 9] and to geoscientific virtual environments [6], to humancentered visualization techniques [5, 3], to usability issues [25] and to 3-D user interface design [2].

Earlier work in investigating terrain meshes (in this case from bathymetry data) via touch was presented in [8]. This system used early versions of several visualization techniques that M4Geo has implemented via shader technology. [11] presented work on integrating surface modeling via force feedback into a geologic 3-D (subsurface) modeling system. Although no longer developed, this work inspired many of the interaction techniques explored in M4-Geo. Aspects of interactive editing large terrain data were discussed in [1]; usability experiments for scientific visualization in immersive virtual environments performed by [28] included interaction with 3-D Mars terrain data via a PDA. [13] presented a speech and gesture driven whole Earth 3D terrain model.

Our work is also related to [Tangible User Interfaces (TUI) for the interactive visualization and manipulation of 3-D terrain models $[17,20]$. These systems deal with a scientific subject matter very similar to M4-Geo's and also address the user's need for more intuitive methods of shaping terrain; however, the physical interface is a shapeable model of "illuminated clay".. Our work is also part of the larger field of haptic visualization $[21,22]$, which is beginning to gain importance. Haptic rendering of 3-D scientific data, either in support of already visually rendered data or without a visual counter part, aims to enhance the users holistic perception and comprehension of complex high-dimensional data, such as computational fluid dynamics (CFD) data [15, 14, 19, and 10].

\subsection{Haptic (Touch-based) Interaction}

The human haptic system incorporates two types of sensory touch information: skin-pressure-based (tactile) feedback and joint/muscle-based force-feedback (kinesthetic). Computer haptics refers to methods for creating haptic impression to a human user via a specialized piece of hardware (haptic interface or display). Much like a 3-D computer graphics system conveys information about virtual (non-existing) 3-D objects to the user via a display, a computer haptics system generates computercontrolled forces to convey the shape, friction, etc. of virtual 3$\mathrm{D}$ objects. To feel the 3-D object, the user needs to be in direct contact with the haptic interface device; the device is, in turn, controlled by haptic rendering algorithms (software). This connection creates a closed loop that continuously exchanges force signals and position signals between the user and the virtual 3-D objects.

For technical reasons the most commonly used type of haptic interface devices used today are point haptic devices, which use only a single point of contact to provide active, kinesthetic feedback but provide no tactile (skin-pressure-based) feedback to the user. In the case of the Sensable Phantom models this single point sits at the end of a small, grounded arm and acts as the tip for a stylus (or pen). The movement of the tip within the haptic workspace is limited to typically 2 to 4 inches on each side. The process of haptically rendering the geometry of a 3-D object composed of triangles, for example a cube, first requires a test to detect if the user guided tip is currently inside the object, if not, no force is generated and the tip's path is not impeded. However if the Phantom's arm detects (tracks) the tip at a position that would be inside the object, a point that lies directly on the surface, the so-called proxy point is calculated and the Phantom's motors generate a counter force so that the tip is pushed towards the proxy. The maximum force generated by a Phantom is only 3-7 Newton, depending on the type of haptic device. Despite the use of discrete "jolts" of force the user's hand is given the impression of feeling the continuous surface of an object that is placed within the haptic 3-D workspace (figure 2 ), because this detect-push-cycle happens at very fast rates, typically $\sim 1000$ times per second. [23] presents an introduction to haptic rendering and provides further details.

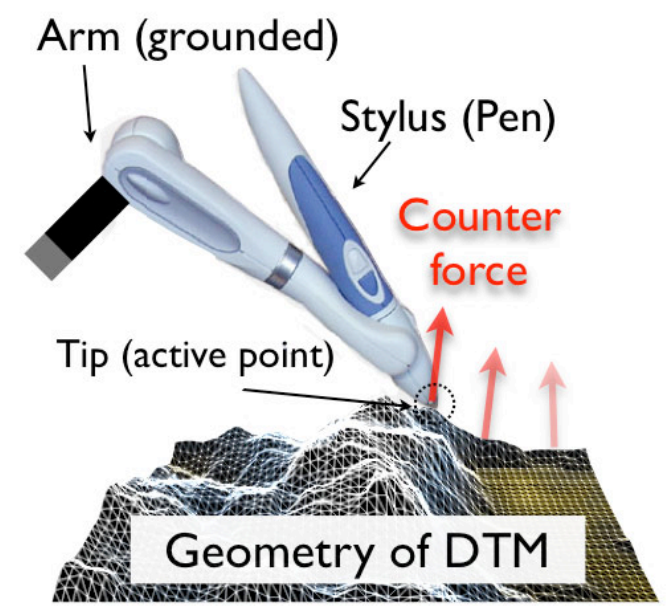

Figure 2. Touching a DTM via a point haptic force feedback device - a fast succession of forces generated at the tip conveys the terrain's geometry to the stylus held by the user

Several haptic properties can be attached to the geometry of such a 3-D object, similar to defining the color of an object for visual rendering. Such haptic properties include stiffness (the softness of an object), static and dynamic friction, attraction effects, and a gray scale image of "micro displacements", similar to bump map textures in visual rendering.

Besides triangle-based objects, poly-lines and points can be rendered via magnetic attraction forces that become active within a certain range and attract the stylus towards the (closest) point. Beyond the rendering of geometrically bounded objects, generalized force field effects can also be used to craft more 
ephemeral expressions, for example, 2-D or 3-D vector field can be rendered by setting the tip's force to the direction and magnitude of data at tip's current location and can facilitate the exploration of higher-dimensional data ([14],[15],[19],[10]). The haptic effects can be mixed together and added to the haptic rendering of 3-D geometric objects. For example, the vectors of water flowing downhill over a terrain surface, could be translated into force vectors and added to the rendering of the surface.

Until recently the cost of haptic force feedback devices was relatively high: even the Phantom Omni, a relatively low end device shown in figure 3 (left) costs around \$2000, larger and more powerful devices cost substantially more. The Novint Falcon (figure 3, right) costs only $\$ 200$ but has a relatively small workspace. It uses a 4-button "knob" instead of a pen for the user to move around and the software would need to compensate for this.

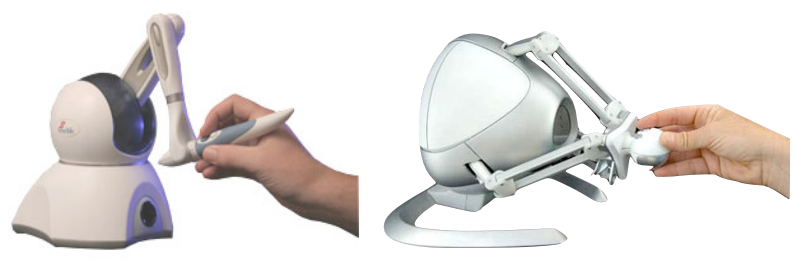

Figure 3. Low-end point force feedback devices: Phantom Omni(left ) and Novint's Falcon (right)

\subsection{Combining 3-D graphics and haptics}

The power of 3-D interactive graphics for 3-D geovisualization is well established. The M4-Geo system uses the Phantom force feedback device as an addition to a 3-D visual environment, using either monoscopic or stereoscopic display technology. This results in what could be called a form of multi-sensory (vision + touch) virtual vnvironment in which the Phantom's stylus provides a virtual tool that is held in the user's dominant hand. In addition, the user's non-dominant hand could be represented by a second Phantom, opening up many new ways for interacting with 3-D objects, some of which we are currently investigating.

As the user moves the Phantom's tip and rotates the stylus around the tip, both are displayed in 3-D relative to the other 3D objects, for example a 3-D terrain model. M4-Geo uses this setup to provide the user with a very intuitive form of egocentric navigation system, where the terrain model is treated as an object that sits in front of the user, and which the user can move and rotate (grasp) while the user's head position remains static. When the user touches the surface and holds down the stylus button, the surface is momentarily attached to the stylus and the user can move and rotate the surface, dropping and re-grasping it as needed. If a second Phantom is available for a second hand, it becomes possible to grasp the surface at two points and to scale it up or down by pulling the two points apart or bringing them closer together (similar to the zoom functionality implemented in multi touch devices such as the iPhone).

Because of the size and mechanical nature of the Phantom's arm, it is advantageous to use stereo graphics and a mirrored display to give the user the impression that the physical stylus of the device is co-located with the virtual stylus and that the visual workspace and the haptic workspace thus overlap spatially (figure 4).

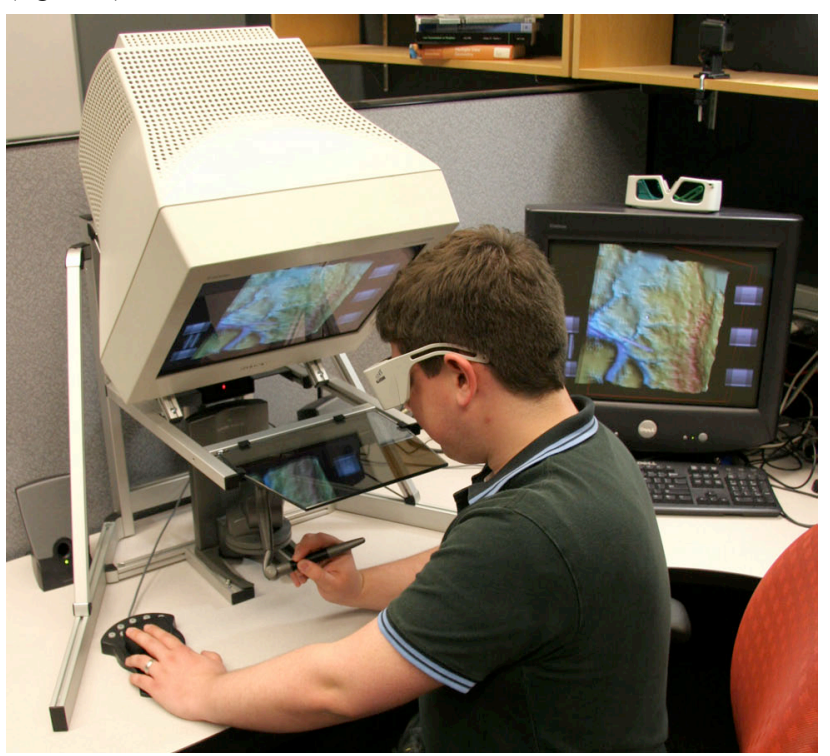

Figure 4. Using a colocated setup where active stereo graphics are displayed onto a mirror, beneath which the phantom is operated

\section{Methods}

\subsection{Software and Hardware used}

The M4-Geo system uses the open source H3D API created by SenseGraphics AB, its current version is 2.0. $\mathrm{H} 3 \mathrm{D}$ is an implementation of the X3D scenegraph API (the successor to VRML) extended for haptic rendering and for haptic interaction via one or more point haptic force feedback devices. H3D supports the SensAble line of Phantoms (Omni, Desktop, Premium), ForceDimension's Delta and Omega devices and Novint's Falcon device. H3D renders a 3-D scene graphically and haptically; the scene's objects have graphical properties such as color, and haptic properties, such as friction. Much like other scenegraph APIs, such as OpenSG, OpenScenegraph, and Performer, H3-D is a high level API that takes care of many complex rendering details and internally converts high-level concepts into calls to lower level haptics APIs (the equivalent to OpenGL for 3D graphics). For example, H3D automatically provides the haptic thread needed for the concurrent haptic rendering and collision detection at rates of $\sim 1000$ frames/second while the scenegraph (and the graphics) are updated at $\sim 30-60$ frames per second. H3D typically uses a point-based haptic proxy model, a sphere-based proxy model is offered in the latest version (2.0).

$\mathrm{H} 3 \mathrm{D}$ provides configuration files to adjust the haptic workspaces' size and position, the display setup, and other parameters to accommodate the different devices and displays. This flexibility allows us to run the M4-Geo software in many different settings. We have used it with a standard mono display (even on a laptop), with an active stereo display (using a NVidia Quadro card and a CRT monitor) and with a passive stereo display (Arisawa P240W LCP display). We have used it with a single Phantom (Omni, Desktop or Premium model) or a Novint 
Falcon attached. We have also explored the use of two Phantom devices together where one Phantom acts as dominant hand for operating a virtual tool and the second Phantom can simultaneously translate and rotate the object as if it were an object held in the users other hand.

$\mathrm{H} 3 \mathrm{D}$ provides a basic viewer (H3DViewer) for viewing and touching the content of a X3D file. X3D retains many elements of the older VRML2 format but uses the XML-style syntax. For example, the text file shown in figure 5 creates a sphere in the center of the haptic workspace with visual and haptic properties. M4-Geo uses this X3D file format to initially load 3-D objects, such as triangle meshes, into scenegraph nodes.

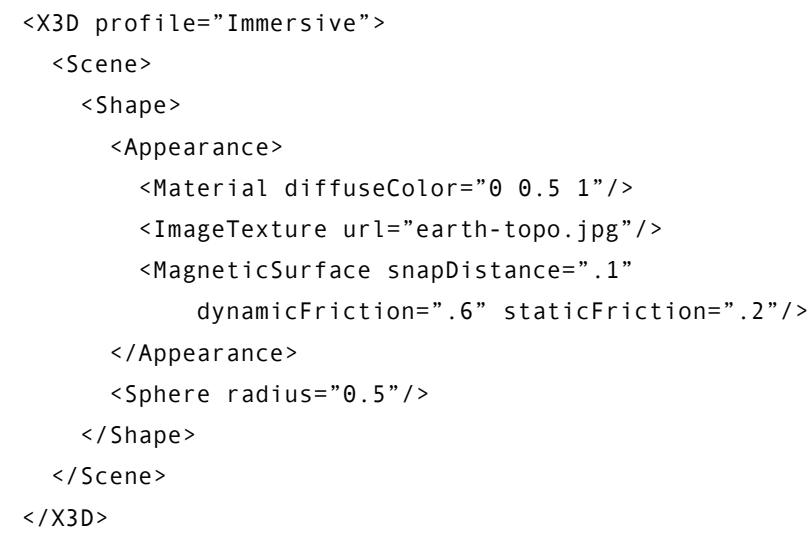

Figure 5. Example of a touch-enhanced X3D file

One of the most powerful aspects of H3D is its ability to use python scripts as part of the scene graph. Python scripts can read or change the attributes of the scenegraph nodes through predefined fields; they are key to implementing complex, event driven user interactions between the haptic device and the scenegraph objects. For example a python script could map the force (pressure) the user applies to a sphere to the visual transparency of the sphere and making it fade away as more and more force is applied.

For more complex or time critical functionality H3D also offers an open source $\mathrm{C}++\mathrm{API}$, which can be used to extend the official H3D scene graph nodes via sub-classing. Coding in $\mathrm{C}++$ also provides access to lower level APIs such as OpenGL and lower-level haptics APIs, and can be used to compile a custom $\mathrm{H} 3 \mathrm{D}$ viewer with additional functionality. For M4-Geo, we created such a custom executable by extending several classes of H3D nodes (such as the indexed triangle set class) to support additional types of manipulation. This includes the addition of a topology database to localize real-time alterations in the mesh connections and the inclusion of an octree spatial partition to improve performance when locating elements within the mesh.

We make extensive use of H3D's ability to incorporate customized 3D graphics routines, called shaders. Shader programs execute directly on the 3D graphics hardware (the GPU, Graphics Processing Unit) and provide substantial increases in flexibility and performance, especially on modern graphics cards. Our shaders are written in the GLSL high level shading language (based on OpenGL) and are integrated into the
H3D scenegraph via shader nodes and routes. This makes it possible for the shader's effects to be directly tied to the Phantom and thus to the user interaction with the data. For example, the stylus orientation in 3D determines the direction of a virtual sun that lights the terrain, this is continuously transmitted to the shader running on the graphics card and changes the 3D illumination of the terrain, similar to hillshading. Slowly reorienting the stylus can bring out minute details of the terrain's morphology that are tied to a prevailing direction. Similarly, a "flashlight" that is connected to the stylus may show certain aspects of the data within its cone, similar to a magic lens. M4-Geo uses several other shader programs to efficiently visualize changes to the terrain as a result of user interaction, for example the visualization of the surface's gradients during its deformation or the application of color directly into the terrain's texture map during the interactive painting process.

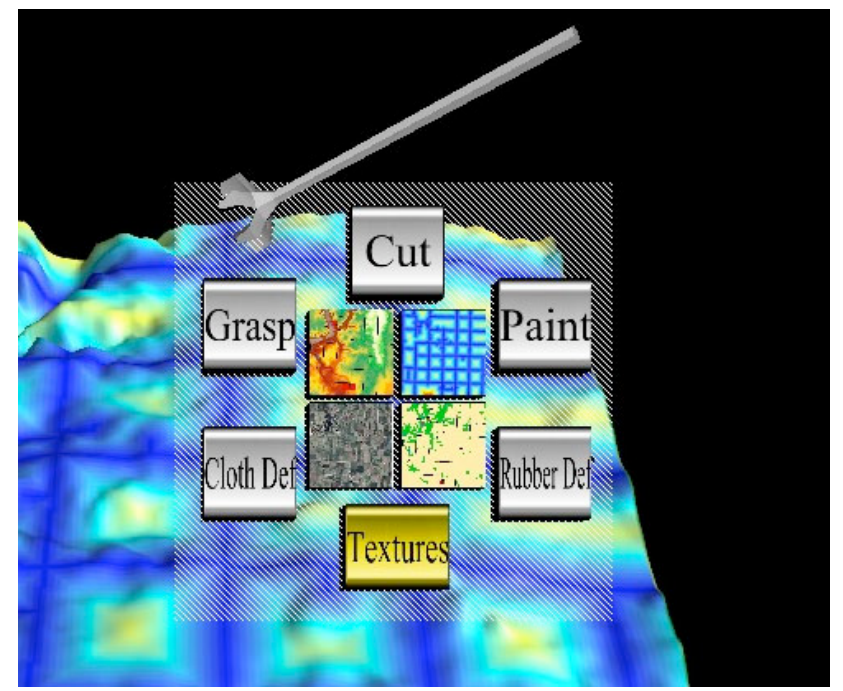

Figure 6. M4-Geo's touchable User Interface panel

We use the phantom's stylus for all interaction tasks, including interactions with the graphical user interface (GUI). The GUI type interactions, like changing the currently active virtual tool, changing the visualization parameters, such as the currently displayed raster or fine tuning the way a tool operates (e.g., changing the size or color of the virtual brush) are performed via a touch sensitive menu panel (figure 6). When activated, it will appear in the 3-D environment close to the position of the stylus and can be hidden again once the user has changed the desired setting, such as selecting a different type of virtual tool. Threedimensional models are used to symbolize the type of the currently active tool, e.g. a brush symbolizes the paint tool, the "claw" shown in figure 6 indicates that the user will grasp the model to move and rotate it.

\subsection{GIS Data preparation}

A digital terrain model (DTM, also called digital elevation model or DEM) refers to a digital representation of a part of the Earth's surface derived from various survey techniques. Its representation in 3-D space is based on a set of 3-D points, either with $\mathrm{x} / \mathrm{y} / \mathrm{z}$-positions (in a Cartesian system) or latitude/longitude/height (in a spherical system). These points are connected into a continuous surface and stored either as the centers of a regular grid or as the nodes of a triangle mesh (or 
TIN). Although using points with positions in 3-D space, a DTM typically does not contain any points with different $\mathrm{z}$-coordinate (height) values but identical $\mathrm{x} / \mathrm{y}$-coordinate, which could represent an "overhang". The typical DTM surfaces are therefore more precisely called 2.5-D surfaces. The process of creating a 3-D object from values contained in a 2-D height field (or height map) by offsetting them up or down in the elevation (z) direction, is sometimes called "rubber sheeting".

Geospatial raster and vector data that is to be used within the M4-Geo system needs to be converted into a X3D file format. We typically start with a raster DTM in ESRI's grid format, values of the raster's cells are used to create the additional "halfdimension". High resolution data for elevation and many other attributes, are now widely available at different raster resolutions, for example from the USGS Seamless Web site [26]. Non-elevation raster data, such as USGS topographic maps, satellite imagery or land use rasters, can be combined with topographic information by draping them over the DTM's geometry (shape). Vector GIS data, such as city outlines, roads, rivers, elevation contours, sample points, etc. may be added to provide visual guidance. To cope with possible differences of projections, extent, and resolutions we first load all the desired raster data as layers into ESRI's ArcGIS and make all the raster data fall into a common area. Additional raster layers may be the result of GIS spatial analysis of the DTM (e.g., slope, azimuth or curvature) or be the result of spatial modeling involving other GIS data (raster or vector data) within the area, for example a view shed raster for several observation points.

Once all the relevant GIS data has been assembled, the DTM is exported from ArcGIS to an ASCII file and then converted into a continuous triangles mesh (a X3D indexed triangle mesh) by a process involving custom written scripts. To prevent geometric distortion, the coordinates of the mesh's vertices are stored in geospatial coordinates (typically UTM). If the terrain data is not stored as a regular grid but rather as a set of irregularly spaced points, as is the case with some forms of LIDAR data, it is first triangulated into a TIN and then converted into a X3D indexed triangle mesh. To make it possible to drape the additional GIS rasters on to the triangle mesh, each of the mesh's vertices is connected to a 2-D location within these images (texture coordinates). We convert these rasters into high resolution images (textures), with sizes typically ranging from 2048 x 2048 pixels to 4096 x 4096 pixels via ArcMap's "export map" function. Besides creating visual texture maps from the rasters, we can also create grayscale images that may be used as haptic texture maps. These haptic textures also draped over the terrain mesh but add certain haptic information at each of the raster's cell locations; for example, a low cost value may translate into low friction, a high cost value into high friction at a spatial location. Alternatively, a small amount of additional force may be used to create the feeling of small depressions (low value) or bumps (high value) on top mesh's geometry, this is called a haptic displacement map. As the user moves the stylus over the terrain, such haptic textures may either augment already displayed (visual) information, or convey additional, non-visual information to the user.

It is possible to include GIS vector data in M4-Geo by either converting it into a raster and then into the texture maps or to create 3-D objects by placing the vector data on top of the terrain. While the raster conversion allows the use of vector data as visual landmarks (e.g., major roads or points representing key cities), the conversion into 3-D permits the use of gravitational haptic force effects. For examples, roads that have been converted in a set of 3-D polylines can be configured to attract the tip with a small force when brought within a certain distance, which can be useful in cases where snapping or computer guidance is desired. The final result of this data preparation procedure is the creation of a single "master" data file that contains the desired terrain mesh as indexed triangle set and any other 3-D geometry within a common 3-D coordinate area. When the M4-Geo system is executed it loads this data file and sets up a series of scaling, rotation and translation operations that fit the data into a roughly $40 \mathrm{~cm}$ cube that is centered around the resting point of the Phantom's tip and display accordingly. If a stereo, co-located setup (figure 4) is used, the cube and it's content will appear to float in front of the user.

\section{Examples of 3-D interactions with geospatial data}

There has been much work during the last decade in the GIS community to move methods for viewing geospatial data from $2 \mathrm{D}$, the electronic equivalent to paper maps, to $3 \mathrm{D}$. Some of these $3 \mathrm{D}$ viewers can also be used with a true parallax based stereo system (i.e., a system that provide slightly different image for each eye), which some consider a hallmark of a virtual environment. For example ESRI's ArcScene/ArcGlobe and Google Earth can be seen as types of 3D visualization applications for GIS data and ArcScene can display this data in different forms of stereo.

While there has been some development to support the 3-D nature of these applications by offering specialized hardware for 3-D navigation, such as the 3DConnection's SpaceNavigator and SpaceExplorer, mouse and keyboard remain the most common devices for 3-D navigation and for interacting with the data as it is displayed in 3-D. This creates a fundamental user interface problem for interactions with 3-D data, including several typical GIS operations. We propose that, even with its technical limitations, point-haptics force feedback devices, like the Phantom, may help solve these problems by providing direct 3-D interaction with geospatial data. Their ability to "display" force may provide substantially more intuitive tools for certain 3-D tasks and permit the users to also use force input to express their intent more efficiently. For example, using the Phantom permits us to create a virtual brush tool in which the amount of paint is tried to the amount of force used during painting.

\subsection{Digitizing line features on a 3-D surface}

Digitizing a polyline or a polygon on a terrain map are common GIS tasks; for example, digitizing a line may be used to create spatial information about the course of a newly planned road or about a geologic fault. The M4-Geo system offers a virtual pen tool for digitizing lines directly onto a 3-D model thus seamlessly combining visual and haptic feedback. The tool's interaction is modeled on the act of drawing on a physical 3-D shape with a pencil. Moving the virtual pencil to a desired location and then clicking the stylus button results in a succession of line segments, similar to digitizing on a 2-D map with a mouse or a digitizing tablet. However, as the 3-D surface may rise and fall between the two points; M4 uses a plane (rectangle) that is oriented in 3-D by the stylus to visualize the rise and fall of the terrain between the two points. In addition to conveying the position of the pencil tool's tip on the terrain surface and with the context of geospatial data draped on the 
surface, the force feedback of the system informs the user when the tip is in contact with the terrain. As the force feedback keeps the tip precisely on the surface mesh, rubbing the tool over the surface conveys its morphology. Feeling these possibly minute bumps and grooves augments the user's visual perception and helps to find the desired location from which a new polyline segment should be added. While the user moves the stylus around on the surface, the predicted intersection of the line with the mesh is constantly shown as a graphical preview.

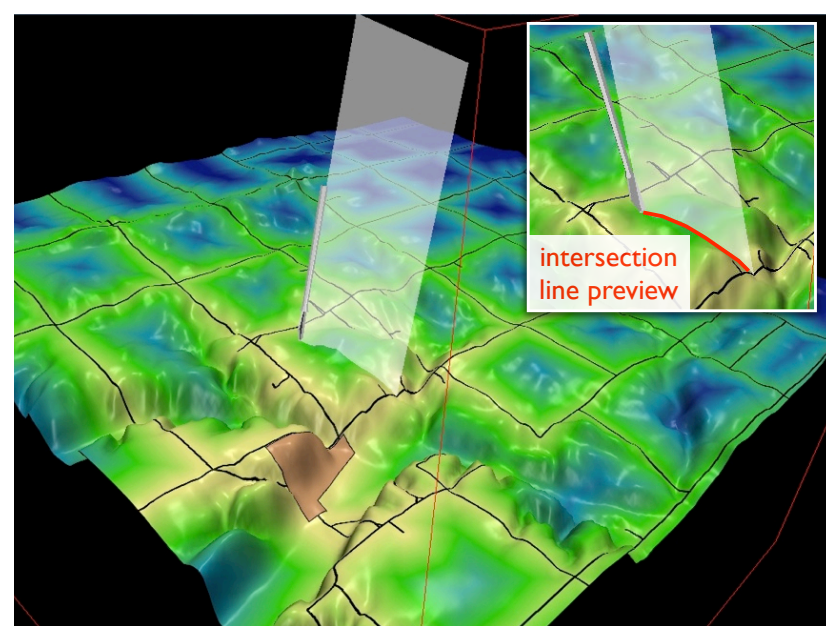

Figure 7. Using the stylus to orient a rectangle that shows the predicted intersection of a new segment with the terrain

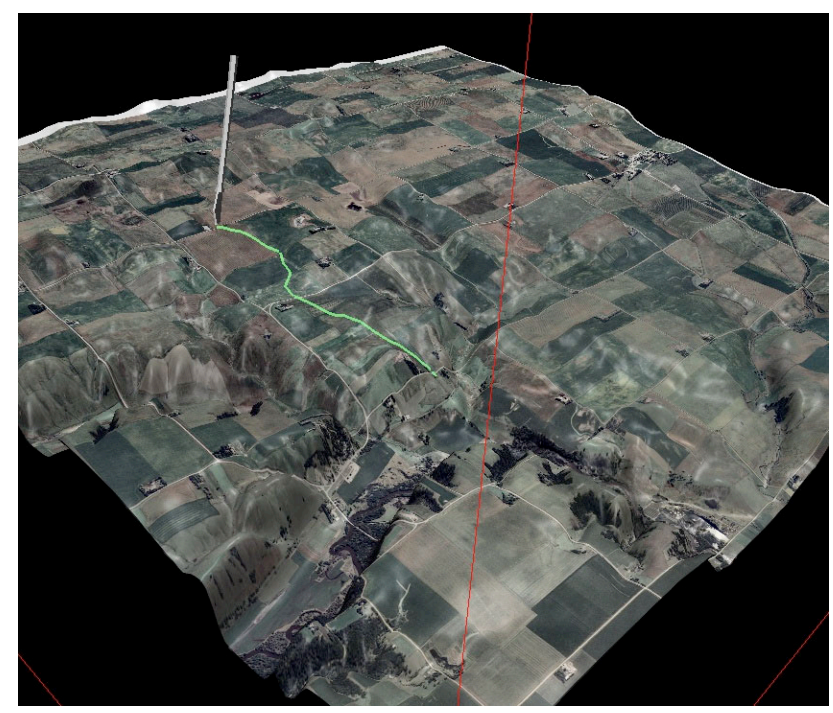

Figure 8. The result of digitizing (draping) a polyline on the mesh

Figure 7 shows the intersection of this line segment with the terrain via a white rectangle, which is anchored at the last point and is slaved to the tip as it moves over the terrain. As the stylus provides a directional vector, the user can change this predicted line in subtle ways by reorienting the stylus around the tip and thus changing the intersection angle of the white rectangle with the terrain (inset of figure 7). Once the user is satisfied with this graphical preview, the new line segment is added to the mesh (Figure 8). In some cases it could be desirable to also convey more information about the touched location during the digitizing process. For example, the user may want to augment the aforementioned haptic feedback about the terrain's shape by also conveying the cost raster, shown draped on the DTM in figure 7 via friction. This provides a secondary stream of strictly local data that may be useful during certain planning tasks, such as digitizing a road while viewing the air photo shown in figure 8 .

\subsection{Touch pressure based spatial selection}

Besides digitizing polylines, which uses a drawing/pencil metaphor, M4-Geo implements the spatial selection of raster data via a painting/brush metaphor. The virtual paint tool can be used to select parts of the terrain by applying a color to a separate selection layer that overlays the other raster data layers (textures), as is shown in figure 9. This selection can later be used to perform a subsequent GIS operation only within its color, similar to a zone in ArcGIS. To give the user more flexibility, the input force is translated into a brush size, i.e., pressing harder against the terrain surface increases the radius of the paint. A preview mode shows this predicted "footprint" in a semi-transparent color, the images in figure 10 (left to right) show the effect of increasing the force applied to the object. When the user is satisfied with the preview, pressing the stylus button begins applying color; this produces a smooth paint trail as the tip is moved over the terrain. In addition to painting, the tool can be switched to an erase mode to remove previously applied paint.

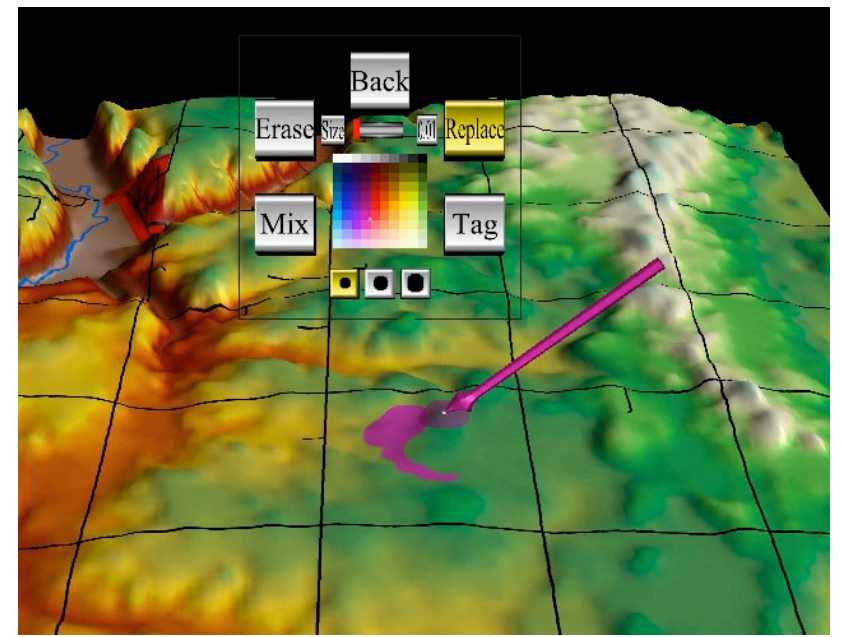

Figure 9. Manual spatial selection of parts of the terrain surface using the interactive paint tool

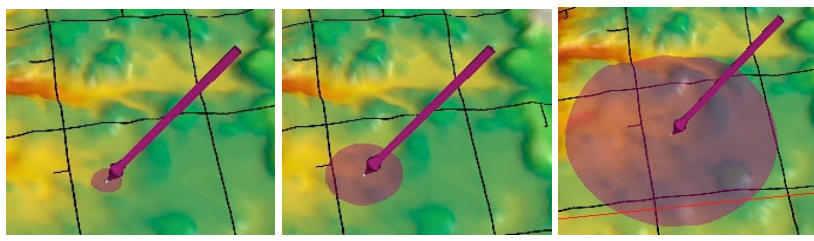

Figure 10. Adjusting the size of the paint tool's brush by varying the force applied to a location on the terrain mesh 


\subsection{Spatial selection via slope based flooding}

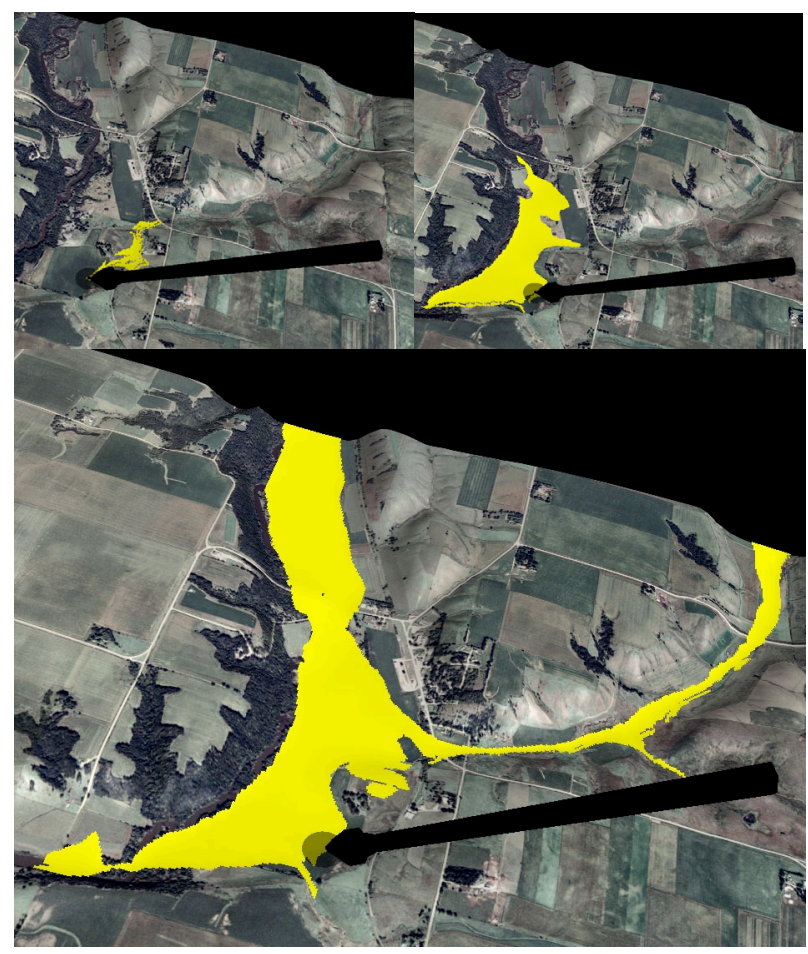

Figure 11. Effects of using the flooding selection tool with increasing amounts of force

This variation of the paint tool selects a continuous region around the tip (yellows pixels in figure 11), depending on the position of the tip and the force applied by the user. Beginning at the tip, a flooding algorithm expands this set of pixels outwards, depending on the terrain's slope beneath each new pixel. The flooding terminates where the slope reaches a certain threshold value. This threshold value is again dependent on the force the user exerts on the terrain, i.e., the harder the tip is pressed onto the terrain, the larger the selected region tends to become.

The effect of increasing the force can be seen in figure 11; the tip's location within a river valley remains constant and the forces increases steadily from the upper left image to the lower image. While the current flooding algorithm is very simplistic it demonstrates how real-time spatial computations can be integrated into touch based interactions. The current algorithm can be used to investigate the flooding potential for locations that might be part of a river's floodplain.

\subsection{Interactive deforming of the terrain surface}

Most of the typical user interactions in 3-D visualization systems relate to changing aspects of the appearance of the data. However, the deformation tool permits the user to affect the terrain's geometry on the vertex level. Figure 12 shows the use of the deformation tool, the user touches the surface at a location (upper-left image), holds down the buttons and moves the tip in any direction.

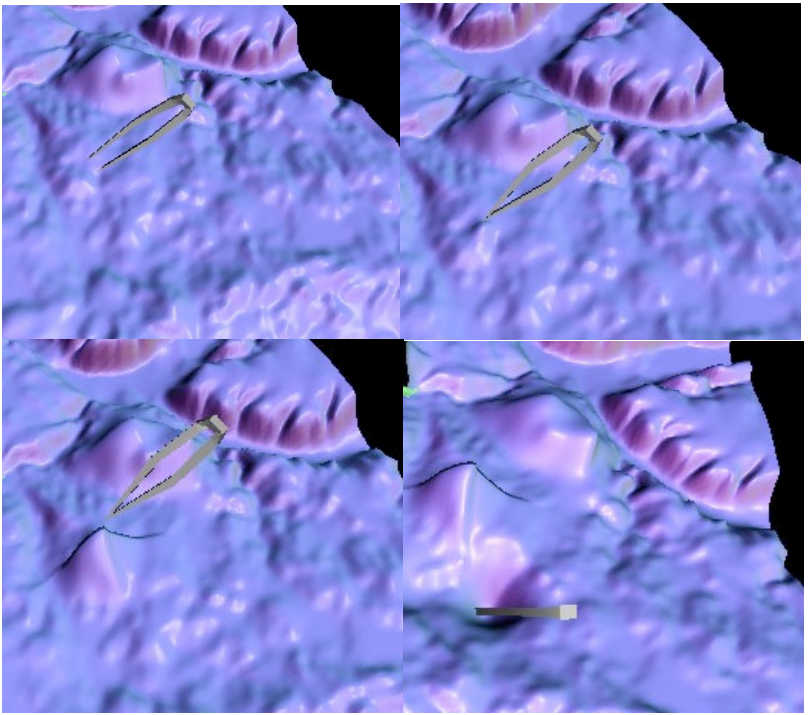

Figure 12. Real time terrain deformation

In the upper-right image, the tip was moved slightly upwards. M4 Geo's implementation of the Generalized Chainmail deformation algorithm $[30,31]$ calculates how far certain vertices of the underlying triangle mesh have to be moved in order to create the geometry of a new hill (lower-left image) or a depression (lower-right image) within the terrain mesh. As the stylus tip continues to move farther away from the initial location, this real-time algorithm will move an increasing number of vertices around in a way that simulates the deformation of a cloth-like material and that distributes the vertices evenly. During the deformation, the terrain's visual appearance is adjusted and the force the user perceives is calculated to indicate the degree of deformation; the farther the tip moves from the origin of the deformation, the larger the deformation becomes, and the larger the counter force that the user feels. The images shown in figure 12 also demonstrate the effect of a special coloring mode, which temporarily colors the terrain according to its direction (normal). This accentuates the terrain's gradients and enhances the user's perception of the details of the terrain's morphology. As it is implemented through a per-pixel shader, it shows the changes to the morphology created by the use of the deformation tool immediately and helps the user adjust the deformation.

The nature of the deformation is defined by several parameters that the chainmail algorithm uses to decide which vertices to move and by how much. These parameters can be used to tweak the deformation behavior, i.e. the type of shape the terrain takes in response to moving the stylus tip around. In M4-Geo, these parameters are applied to the vertices of the mesh and thus can be thought of as defining the material properties of the mesh with regard to deformation. For example, moving the tip when deforming a conceptually "soft" terrain results in a steep, "pointy" hill, whereas the identical tip movement for deforming a conceptually "hard" terrain would result in rounder hill. While this degree of softness is initially set globally for all mesh vertices to a medium-soft material, the user can re-define the mesh's type of material (very hard to very soft) locally by painting a special material color onto the terrain. 


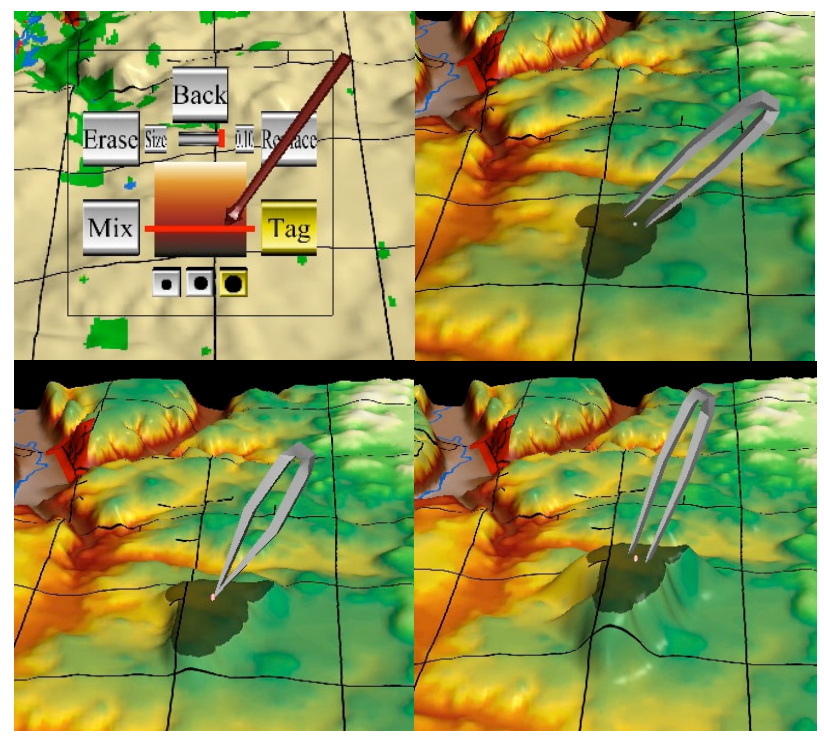

Figure 13. Constrained terrain deformation by first painting parts of the terrains to become harder (black)

The upper left image in figure 13 shows the selection of this material color from a black-red-white color ramp, which was chosen as it mimics the color of iron during heating; black suggest cold and therefore impossible to deform, red and finally white suggest higher temperatures and hence material that is increasingly soft and malleable. The black material color can thus be used to "freeze" parts of the terrain and to prevent any deformation. The images shown in Figure 13 (upper right, lower left, lower right) demonstrate the effect of painting parts of the terrain with this black material color and then moving a point inside this black area upwards to create a hill. Unlike the deformations shown in figure 12, the morphology of the terrain painted in black is preserved. Because the digitizing described earlier integrates the topology of its lines into the mesh, they can be used as break lines that limit the spatial extent of the deformation process.

The combination of assigning specific deformation property to certain parts of the terrain (via manually painting or via the flooding tool) and then guiding the deformation of these parts interactively with the support of force feedback provides a radically different way of modifying terrain in the context of digital landscape design and geotechnical planning.

\section{CONCLUSIONS \& FUTURE WORK}

We have presented M4-Geo, a prototype software system that implements several examples of touch-enabled 3-D interactions with geospatial data. The system is written with the open source H3D API and uses a point haptics force feedback device as a virtual fingertip for the multi-sensory exploration of terrain data and raster data draped onto its geometry. The system's interactions focus is initially on direct GIS interaction tasks, where the use of a 3-D force feedback device promises significant advantages over the use of mouse and keyboard. These tasks include digitizing of line data, spatial selection via painting and flooding, and real-time deformation of surface meshes. We have conducted an informal evaluation of these tasks with local domain experts, they feedback has been very positive and indicates that a larger multi-sensory GIS system can provide improvements over current methods in the areas of landscape architecture and geotechnical engineering. For example, a landscape architect could create a 3D digital model for a new golf course; or a geotechnical engineer could explore terrain modification for the prevention of local flooding. Currently, the process of assembling and transforming the different types of geospatial data still requires a chain of import/export procedures and handwritten scripts. This process needs to be simplified and should be as automated as possible to widen the number of potential users. Ideally, it should be possible to export GIS layers from GIS systems (such as ArcGIS or GRASS) into X3D/H3D scene, similar to the VRML export offered in ArcScene.

The implementation of a dual-handed system, in which two Phantoms are used, is in the final stages of testing. Preliminary testing shows that, at the very least, the second hand is useful to seamlessly grasp, reposition and rotate the terrain while it is manipulated by the virtual tool; however, there are also several intriguing potential uses of two tools together; for example, one hand could deform the terrain while the other hand changes the deformation properties with a "blow torch" tool, that can soften (or harden) parts of the area under deformation. The use of two phantoms may also provide interesting possibilities for multiuser interaction and for the collaborative modification of terrain. A more formal evaluation of certain usability aspects of the system using students is currently underway.

We have also explored the addition of sound as an additional visualization modality. Similar to the communication of data values via friction, a series of sounds can be used to convey data about the currently touched location, for example its elevation. Preliminary results suggest that the changes of pitch in such an elevation melody created by moving the tip over the terrain can help the detection of certain terrain features.

While our research currently focuses on the areas of data exploration, there are several other areas of application into which the system could extend to. One such area may be the multimodal presentation of geospatial information to visually impaired students [7]. The use as an educational tool in courses teaching geomorphology has also been suggested.

A variation of the M4 system may become useful for exploring high-dimensional (multi attribute) data and non-spatial data in 3D information visualization context [27], where it could improve the selection of subsets of data via coloring and modifying the way the data is visualized (attribute-based distortion). Force effects could be added to augment the user's knowledge about the data, e.g., magnetic force effects could be used to convey data relationships (connections) between data elements and motion (vibration) could be used to convey uncertainty.

\section{ACKOWLEDGMENTS}

We acknowledge the helpful feedback and suggestions given by the anonymous reviewers. Part of the work was funded by NSF grant IIS- 534681 


\section{REFERENCES}

[1] Atlan, S, and M Garland. Interactive Multiresolution Editing and Display of Large Terrains (2006) Computer Graphics Forum 25.2, p.211-23.

[2] Bowman, D. A., Kruijff, E., LaViola, J. J., and Poupyrev, I. 2001. An Introduction to 3-D User Interface Design (2001) Presence: Teleoper. Virtual Environ. Vol. 10/1

[3] de Haan, G, M Koutek, and FH Post. (2002) "Towards Intuitive Exploration Tools for Data Visualization in Vr," Proceedings of the ACM symposium on Virtual reality software and technology 2002. P.105-112.

[4] Döllner, Jürgen (2005) Geovisualization and Real-Time 3D Computer Graphics, in Exploring geovisualization / ed. by J. Dykes, A.M. MacEachren and M.J. Kraak. Elsevier, 2005 p. 343-366.,

[5] Fikkert, W., T. Bierz, M. D'Ambros, and T.J. Jankun-Kelly. Interacting with Visualizations (2007) In: Human-Centered Visualization Environments, volume 4417 of Lecture Notes in Computer Science. Vol. 4417, chapter 3, p. 257-294.

[6] Fröhlich, B., S.Barrass, B. Zehner, J.Plate and M.Goebel (1999) Exploring GeoScience Data in Virtual Environments, Proceedings of IEEE Visualization 1999

[7] Golledge, R, Rice, M, Jacobson R (2006) Multimodal interfaces for representing and accessing geospatial information. In Frontiers of Geographic Information Technology, p. 181-208

[8] Harding, C., Ioannis A. Kakadiaris, John F. Casey, R. Bowen Loftin (2002) A multi-sensory system for the investigation of geoscientific data, Computers \& Graphics, Volume 26, Issue 2, p. 259-269.

[9] Hay R. J. (2003) Visualisation and Presentation of Three Dimensional Geoscience Information (2003) In Proceedings of 21st International Cartographic Conference 2003.

[10] Ikits, M., Brederson, J. D., Hansen, C. D., and Johnson, C. R. (2003) A Constraint-Based Technique for Haptic Volume Exploration. In Proceedings IEEE Visualization 2003

[11] Kowalik, W. (2001) "3D Device Interaction with. Force Feedback” in: Gocad 3DH, 2001.

[12] Kraak, M.-J., MacEachren, A.M. Geovisualization and GIScience (2005) Cartography and Geographic Information Science, Vol. 32, p. 67-68

[13] Krum, D.M., O. Omoteso, W. Ribarsky, T. Starner, and L.F. Hodges. Evaluation of a Multimodal Interface for 3D Terrain Visualization (2002) in Proc. of IEEE Visualization 2002

[14] Lawrence, D.A.; Pao, L.Y.; Lee, C.D.; Novoselov, R.Y., "Synergistic visual/haptic rendering modes for scientific visualization," (2004) Computer Graphics and Applications, IEEE , vol.24, no.6, pp. 22-30

[15] Lundin, K., M. Cooper, A. Ynnerman: Enabling Design and Interactive Selection of Haptic Modes, Virtual Reality, Volume 11, Number 1 / March, 2007

[16] MacEachren, A. Kraak, M. 2001 Research challenges in geovisualization. Cartography and Geographic Information Science 28, p.3-12
[17] Mitasova, H, L Mitas, C Ratti, H Ishii, J Alonso, R Harmon. Real-Time Landscape Model Interaction Using a Tangible Geospatial Modeling Environment (2006) Computer Graphics and Applications, IEEE 26.4, p.55-63.

[18] Nöllenburg, M. (2007) Geographic visualization. In: HumanCentered Visualization Environments, volume 4417 of Lecture Notes in Computer Science. Vol. 4417, chapter 6, pages 257294. Springer-Verlag, 2007

[19] Qi, W. (2006) Geometry based haptic interaction with scientific data. In Proceedings of the 2006 ACM international Conference on Virtual Reality Continuum and Its Applications (Hong Kong, China). VRCIA '06. ACM, New York, NY, 401404.

[20] Ratti C., Wang Y., Ishii H., Piper B., Frenchman D., (2004), "Tangible User Interfaces (TUIs): a novel paradigm for GIS", TGIS Transactions in GIS, 8:4, pp. 487-421, ISSN 1361-1682

[21] Roberts, J.C., Sabrina Panëels, Where are we with Haptic Visualization? (2007) Second Joint EuroHaptics Conference and Symposium on Haptic Interfaces for Virtual Environment and Teleoperator Systems, Tsukuba, Japan p. 316-320

[22] Roberts, J.C.; Franklin, K., "Haptic glyphs (hlyphs) - structured haptic objects for haptic visualization (2005) Eurohaptics Conference, 2005 and Symposium on Haptic Interfaces for Virtual Environment and Teleoperator Systems, 2005. pp. 369374, 18-20 March 2005

[23] Salisbury, K.; Conti, F.; Barbagli, F. (2004) "Haptic rendering: introductory concepts," Computer Graphics and Applications, IEEE , vol.24, no.2, pp. 24-32, March-April 2004

[24] Sallnäs, E., Rassmus-Gröhn, K., and Sjöström, C. (2000). Supporting presence in collaborative environments by haptic force feedback. ACM Trans. Comput.-Hum. Interact. 7, 4 (Dec. 2000), 461-476

[25] Slocum, T.A., C. Blok, B. Jiang, A. Koussoulakou, D. R. Montello, S. Fuhrmann, and N. R. Hedley (2001) "Cognitive and Usability Issues in Geovisualization," Cartography and Geographic Information Science, vol. 28, pp. 61-75, 2001

[26] USGS Seamless web site: http://seamless.usgs.gov/

[27] Ward, M., and J. Yang (2004) Interaction spaces in data and information visualization. Proc. Joint Eurographics - IEEE TCVG Symposium on Visualization, pp. 137-146 (May, 2004).

[28] van Dam, A., Laidlaw, D. H. and Simpson, R. M. Experiments in Immersive Virtual Reality for Scientific Visualization (2002) Computer \& Graphics 26, p.535-555

[29] Wood, Jo, Sabine Kirschenbauer, Jurgen Dollner, Adriano Lopes, and Lars Bodum. (2005) "Using 3D in Visualization." In Exploring Geovisualization, edited by Jason Dykes, Alan M. MacEachren, and Menno-Jan Kraak, 295-312. Oxford: Elsevier Ltd, 2005.

[30] Gibson, S. (1997) 3Dchainmail: a fast algorithm for deforming volumetric objects. In Proceedings of Symposium on Interactive 3D Graphics, pages 149--154, 1997. MERL-TR-9719 November 1997

[31] Li, Y. and Brodlie, K. (2003) Soft object modeling with generalised chainmail, Computer Graphics Forum, 22(4): pp. $717-7$ 